\title{
La Paz en Práctica: caja de herramientas con enfoque psicosocial para la Jurisdicción Especial para la Paz*
}

\author{
Putting Peace into Practice: A Toolbox with a Psychosocial Focus \\ for the Special Jurisdiction for Peace
}

Paula Andrea Ruiz Álvarez**

Universidad Santo Tomás, Bogotá, Colombia https://orcid.org/0000-0001-9857-4405

Sergio Daniel Arrieta Vera ${ }^{* * *}$ Universidad de La Sabana, Chía, Colombia https://orcid.org/0000-0002-4022-1495

Aida Milena Cabrera Lozano ${ }^{* * * *}$

Universidad Santo Tomás, Bogotá, Colombia https://orcid.org/0000-0002-4377-2516

Martha Patricia Vaca Vaca ${ }^{* * * * *}$

Universidad de La Sabana, Chía, Colombia https://orcid.org/0000-0003-3998-3318

Artículo de investigación

Fecha de recepción: 09 de septiembre de 2019

Fecha de aceptación: 22 de octubre de 2019

* Este artículo de investigación es uno de los resultados del proyecto de Prácticas en Psicología Social, realizado en la Jurisdicción Especial para la Paz (JEP), para optar por el título académico de psicólogos en la Universidad Santo Tomás y en la Universidad La Sabana.

** Estudiante de Psicología de décimo semestre de la Universidad Santo Tomás, sede Bogotá, Colombia. Correo electrónico: paularuiza@usantotomas.edu.co

*** Comunicador social y periodista, y psicólogo de la Universidad de La Sabana. Correo electrónico: sergioarve@ unisabana.edu.co

**** Magíster en Desarrollo Educativo y Social, y docente investigadora de la Universidad Santo Tomás, sede Bogotá. Correo electrónico: aidacabrera@usantotomas.edu.co

***** Magíster en Psicología Social Comunitaria y docente investigadora de la Universidad de La Sabana, Chía, Colombia. Correo electrónico: patricia.vaca@unisabana.edu.co 


\section{Para citar este artículo}

Ruiz Álvarez, P. A., Arrieta Vera, S. D., Cabrera Lozano, A. M. y Vaca Vaca, M. P. (2020). La Paz en Práctica: caja de herramientas con enfoque psicosocial para la Jurisdicción Especial para la Paz. Campos en Ciencias Sociales, 8(1), 61-84. DoI: https://doi.org/10.15332/25006681/5716

\section{RESUMEN}

Para las entidades que hacen parte del Sistema Integral de Verdad, Justicia, Reparación y No Repetición (SIVJRNR), es necesaria la construcción de propuestas metodológicas que contribuyan a la satisfacción de los derechos de las víctimas del conflicto armado en Colombia. En respuesta a dicha necesidad, este trabajo — producto de una experiencia de práctica profesional en psicología social — presenta una caja de herramientas orientada a apoyar metodológicamente las labores del Departamento de Atención a Víctimas (DAv) de la Jurisdicción Especial para la Paz (JEP). A través de ella se favorecerá la construcción de instrumentos para los espacios de divulgación, de formación y de acompañamiento psicojurídico, dirigidos a las víctimas que participan en la JEP. Este proyecto fue diseñado a partir de los postulados del construccionismo social, y de la psicología sistémica, social, comunitaria y de la liberación, así como de la educación popular y del enfoque didáctico. Se elaboró desde una mirada cualitativa, con base en el diseño de las metodologías participativas y mediante el uso de técnicas investigativas de revisión documental, observación participante, sistematización de experiencias y análisis categorial. Como resultado, se elaboraron seis herramientas metodológicas, que responden a las necesidades del DAV, a algunos enfoques diferenciales y a los principios éticos con enfoque psicosocial, los cuales se exponen en el desarrollo del presente artículo.

Palabras clave: psicología, caja de herramientas, construcción de paz, JEP, metodologías participativas. 


\section{Abstract}

It is necessary for the entities that constitute the Integral System of Truth, Justice, Reparation and Non-repetition (Sistema Integral de Verdad, Justicia, Reparación y No Repetición - SIVJRNR), to create methodological strategies in order to advocate for the claiming of the rights of the victims of the Colombian armed conflict. Responding to this need, this project — which is the product of a Social Psychology internship experience- presents a 'toolbox' whose main goal is to provide methodological support the work of the Victims Care Department (Departamento de Atención a Victimas - DAv) of the Tribunal of Transitional Justice in Colombia (Jurisdicción Especial para la Paz-JEP). This toolbox is meant to satisfy the need to elaborate methodological instruments for the divulgation, training, and psycho-legal support processes set up for the victims participating in the JEP. This project was designed based on theoretical elements of the Social Constructionism paradigm and of Systemic Psychology, Community-social Psychology, as Liberation Psychology, as well as popular education theory and didacticism. The 'toolbox' was created from a qualitative perspective, using participatory methodologies and research techniques of documentary review, systematization of experiences and categories analysis. As a final result, six techniques were elaborated, techniques which respond to the needs of the Victims Care Department, and take into account differential approaches and ethical principles that are described throughout the paper.

Keywords: psychology, toolbox, peace-building, JEP, participatory methodologies. 


\section{INTRODUCCIÓN}

El recientemente creado Sistema Integral de Verdad, Justicia, Reparación y No Repetición (SIVJRNR) tiene como objetivo contribuir a la satisfacción de los derechos de las víctimas a la verdad y a la justicia, así como aportar a la convivencia, la reconciliación y la no repetición del conflicto armado en Colombia, por medio de mecanismos extrajudiciales — la Unidad de Búsqueda de Personas dadas por Desaparecidas (UBPD) y la Comisión para el Esclarecimiento de la Verdad (CEv) y un mecanismo judicial, la Jurisdicción Especial para la Paz (Jep) (Gobierno de Colombia y Farc-EP, 2016). En ese sentido, la JEP es el organismo llamado a investigar y juzgar los delitos cometidos en el marco del conflicto, con el propósito de ofrecer verdad, justicia y contribuir a la reparación. Por eso, para la JEP, así como para el resto de las entidades del SIVJRNR, es indispensable garantizar la participación de las víctimas en todas sus instancias y procesos (Alto Comisionado para la Paz, 2016).

Para la JEP, las víctimas del conflicto armado son el centro de todas las acciones que se llevan a cabo, de manera que el rol de ellas es relevante y protagónico en todas las diligencias judiciales. Por esto, para promover la participación efectiva de las víctimas en la JEP y satisfacer sus derechos a la verdad y a la justicia, en la entidad se han creado distintos departamentos, adscritos a la Subsecretaría Ejecutiva de la institución, entre los cuales está el Departamento de Atención a Víctimas (DAV). Esta unidad es el área encargada de garantizar la participación efectiva de las víctimas en todos los procesos que se adelanten, es decir, de brindar asesoría jurídica y apoyo psicosocial a las víctimas del conflicto que participen en distintos procesos, así como llevar a cabo acciones de difusión, capacitación y pedagogía a organizaciones de víctimas u otras poblaciones que sean de interés del DAV, con un enfoque territorial, diferencial y psicosocial (Ana María Ramírez López, comunicación personal, 18 de febrero del 2019).

En el marco de sus funciones, existe la necesidad de construir metodologías que permitan divulgar información de la JEP, así como fortalecer las capacidades jurídicas y psicosociales de las víctimas, de modo que se pueda garantizar su participación. Por lo tanto, desde la experiencia en prácticas, se buscó aportar en la construcción de una caja de herramientas que contribuyera a la labor misional del DAv. 
Construir herramientas metodológicas que contribuyan a la labor misional del DAV fue pertinente y necesario, teniendo en cuenta las necesidades sociales que exigen la reglamentación y la materialización de este tipo de acciones. Desde el punto de vista legal, garantizar la participación de las víctimas está consagrado como uno de los principios y deberes de todas las entidades que integran el SIVJRNR. Esta participación, de la cual se habla en el punto 5 del Acuerdo de Paz (Gobierno de Colombia y Farc-EP, 2016), está reglada en la Ley 1957 o Ley Estatutaria para la Administración de Justicia en la JEP (2019), la cual especifica que "el Estado tomará las medidas necesarias para asegurar, con perspectiva étnica y cultural, el acceso a información, la asistencia técnica y psicosocial, y la protección de las víctimas ocasionadas por las conductas que se examinarán en la JEP” (art. 14).

Por esta razón, el aporte de técnicas metodológicas con un enfoque pedagógico y psicosocial que ayuden a materializar la participación efectiva de las víctimas en la JEP contribuye al cumplimiento de uno de los deberes que la Jurisdicción tiene con las víctimas. Además, este proyecto que se presenta aquí es pertinente por la importancia social que tiene la participación de las víctimas en los procesos que buscan la satisfacción de sus derechos a la verdad y a la justicia. De hecho, según el Acuerdo Final, se establece que:

La discusión sobre la satisfacción de los derechos de las víctimas de graves violaciones de derechos humanos e infracciones al Derecho Internacional Humanitario con ocasión del conflicto requiere necesariamente de la participación de las víctimas, por diferentes medios y en diferentes momentos. (Gobierno de Colombia y Farc-EP, 2016, p. 124)

Así, las herramientas metodológicas propuestas contribuyen al fortalecimiento de las capacidades de las víctimas para hacer efectiva su participación en la JEP. En ese sentido, el ejercicio de construir herramientas metodológicas atiende a las necesidades del DAV, que consisten en materializar en acciones concretas — jornadas de formación y talleres con organizaciones de víctimas — diseñadas para ser inclusivas, dialógicas y participativas. 
Teniendo en cuenta lo anterior, este trabajo tuvo como propósito construir una caja de herramientas para apoyar el acompańamiento psicojurídico a las víctimas, así a como los procesos de difusión, formación y capacitación sobre la JEP y los mecanismos que tienen las víctimas para participar en ella. Para esto, se identificaron los recursos, las necesidades y los actores de interés para el DAV; se destacaron las voces de los actores de interés del DAV en la construcción de herramientas metodológicas, y, por último, se diseñaron y ejecutar estrategias metodológicas para fortalecer las capacidades de participación de las víctimas en la Jurisdicción.

Para la construcción de tales herramientas metodológicas fue necesaria una mirada teórica que permitiera identificar conceptos transversales para las estrategias metodológicas propuestas, en especial cuando estas están orientadas al trabajo con víctimas del conflicto armado, lo que exige un enfoque diferencial, territorial y psicosocial. La perspectiva epistemológica se orientó desde el construccionismo social y la disciplinar, desde la psicología sistémica, la psicología social comunitaria y la psicología de la liberación. Además, se alimentó la construcción teórica a partir de elementos interdisciplinares propios de la educación popular, específicamente de la pedagogía del oprimido y de conceptos de la didáctica.

Desde el construccionismo social, se entiende que la realidad es una construcción social mediada por el lenguaje, comprendido no solamente por su función de transmisión (Agudelo y Estrada, 2012). Esta visión propone que el conocimiento y la ciencia deben tener un sentido social de transformación, que involucre tanto valores personales como sociales, lo cual permite construir un conocimiento por y para el otro. Según Kenneth Gergen (citado por Agudelo y Estrada, 2012), las fuentes de las acciones humanas se ubican en las relaciones y en el funcionamiento individual dentro de los intercambios comunitarios, lo cual implica que, en esta construcción de realidades, las particularidades individuales tienen una incidencia en la esfera social, y viceversa.

Esta perspectiva resulta pertinente para la construcción de una caja de herramientas puesto que, desde el punto de vista metodológico, invita al manejo atento del lenguaje en las estrategias propuestas, bajo el entendimiento de que este construye realidades. 
Además, estas estrategias permiten la transformación de realidades, a partir de una relación dialógica con los participantes, tras un reconocimiento contextual e histórico. Por tanto, desde esta mirada epistemológica, es relevante mencionar qué perspectivas teóricas de la psicología, con miradas sociocomunitarias, orientaron la construcción de las metodologías.

En primer lugar, esta caja de herramientas utiliza aportes del enfoque sistémico, el cual permite la comprensión relacional de los sistemas humanos. Lo sistémico permite aprehender y ver el mundo desde opciones que expliquen una lectura de la realidad en términos de interacciones, relaciones y roles asignados a los miembros de los mismos sistemas. Esta perspectiva invita a evitar el pensamiento lineal en el análisis de problemas; en cambio, prefiere que estos sean tratados desde una causalidad circular, en la que se evidencia una interrelación de varios elementos (Stanton y Welsh, 2012).

Desde esta perspectiva, los fenómenos humanos pueden entenderse como acciones que se expresan en narraciones humanas, que existen en el lenguaje y son particulares del contexto narrativo del que surgen significados (Anderson, 1997). En consecuencia, el cambio se da a través de la creación de diálogos en el que emergen nuevas narrativas.

Se observa que, en el desarrollo profesional del psicólogo y en general, se pueden evidenciar lógicas jerárquicas verticalizadas. En ese sentido, desde lo sistémico, el reto es ver la humildad no como sinónimo de debilidad, a partir de una perspectiva que les permita a los profesionales no posicionarse "por encima" de las comunidades, sino construir en conjunto con ellas, lo que establece una relación más heterárquica. Lo anterior implica reconocer la riqueza de la diversidad y el saber del otro, de manera que se entiende la comunidad o el consultante como "experto" en su realidad. Esta mirada aporta a la caja de herramientas en cuanto que orienta la relación de los profesionales que van a llevar a cabo metodologías, a través de premisas que se deben tener en cuenta en las acciones psicosociales, que implican la comprensión del otro a través del entendimiento de su contexto y de sus relaciones. 
Además, la perspectiva sistémica enriquece el análisis sobre la complejidad del fenómeno, al ampliar y profundizar los impactos sociales desde una lectura interrelacional, lo que posibilita un análisis amplio del contexto de las comunidades a las cuales van dirigidas nuestras herramientas. Lo anterior invita a incorporar perspectivas teóricas que hayan surgido a partir de los fenómenos y necesidades del contexto local, en este caso, el latinoamericano.

En relación con lo anterior, otra corriente teórica que orientó las herramientas que se construyeron es la psicología social comunitaria que, al igual que todas las corrientes de la psicología de América Latina, se entiende como una psicología que busca el cambio social, a partir del carácter político que tiene la acción comunitaria (Montero, 2004). A pesar de que para Maritza Montero (2003) la psicología social comunitaria no es una corriente teórica basada en una metodología o teoría determinada, muchos de sus pilares teóricos están orientados a la construcción de saberes, métodos y acciones que ofrezcan soluciones a problemas sociales locales.

La psicología social comunitaria es una visión psicológica relevante para proyectos cuyo objetivo sea el trabajo dialógico con víctimas de violencia sociopolítica, puesto que cuestiona la visión de poder en las relaciones y en las ciencias sociales. Le apuesta al establecimiento de relaciones transdisciplinares que involucren el conocimiento popular y aporten a la transformación social, tras el desarrollo de conciencia social, el fortalecimiento y el respeto a la autonomía de las comunidades. Así, esta mirada constantemente integra de forma participativa los saberes comunitarios en sus acciones, con lo que construye una teoría que se retroalimenta con la práctica diaria y viceversa (Montero, 2004). Por lo tanto, es imperativa la incorporación de conocimientos populares, lo cual implica tener como fin la construcción de espacios comunitarios en los que las relaciones de poder sean dialógicas (Montero, 2004).

Este objetivo de destacar los saberes populares es compartido y potencializado, desde la psicología de la liberación (Montero, 2004). Esta perspectiva teórica propuesta por Ignacio Martín-Baró sostiene que todos los actores sociales involucrados en relaciones de opresión construyen el accionar de esta psicología, la cual busca contribuir significativamente a dar respuesta a los problemas cruciales de los pueblos 
latinoamericanos (Trotta, 1998). Para ello, Martín-Baró da cuenta de tres tareas urgentes de la psicología latinoamericana: la primera, contribuir en la recuperación de memoria histórica de los pueblos; la segunda, desideologizar el sentido común y la experiencia cotidiana de la violencia, $\mathrm{y}$, por último, potenciar las virtudes populares (Trotta, 1998).

Estos pilares de acción buscan que la psicología descentre su estatus científico y se ocupe de estar al servicio de la transformación de realidades sociales, así como de aportar soluciones a problemáticas locales, desde una praxis que priorice la transformación de la realidad. Además, desde esta perspectiva, la acción psicosocial se entiende como "como la conjunción entre lo psicológico y lo social, permitiendo de esta forma una comprensión holística de las situaciones humanas entendidas como un todo" (Martín-Baró, 1990, citado por Medina, Layne, Galeano y Lozada, 2007, p. 180). De esta manera, se comprende la relación entre lo intersubjetivo y lo intrasubjetivo, en la cual el contexto social tiene una influencia directa en lo individual, por ejemplo, en el caso de cómo la guerra genera un impacto psicosocial (Martín-Baró, 1990, citado en Medina et al., 2007).

Estas corrientes de la psicología aportan varios elementos a la caja de herramientas. Primero, invitan a la comprensión local del contexto problemático al que se enfrentan las comunidades que reciben estas metodologías, desde una mirada interrelacional. En segundo lugar, promueven la incorporación de saberes populares durante la implementación de las herramientas, lo que estimula el uso de métodos verdaderamente participativos. En tercer lugar, permiten reconocer la necesidad de que, en el diseño, la ejecución y la evaluación de estas estrategias, se tenga como fin contribuir al fortalecimiento de capacidades —en nuestro caso, jurídicas y psicosociales - de modo que las comunidades logren transformar en alguna medida su realidad social, a través de su participación en la Jurisdicción.

Por otro lado, ya que parte del propósito de la caja de herramientas es que las actividades diseñadas aporten a los procesos de difusión y formación, es necesario incluir aspectos relacionados con la pedagogía crítica en esta fundamentación teórica, en la medida en que contribuyen a la generación de espacios y técnicas metodológicas 
que permiten construir saberes en conjunto con las comunidades. En ese sentido, se incluyen aspectos relacionados con la educación popular y la didáctica.

En la teoría de la educación popular, propuesta por Paulo Freire, se considera que las prácticas educativas deben transformarse para pasar de una educación bancaria - en la cual las prácticas educativas son tradicionales y consisten en la transmisión de conocimiento del educador al educando - a una educación popular, en la cual el educando y el educador construyen conocimiento (Freire, 1968; Ocampo, 2008). Ese proceso se construye a través de una relación dialógica, una visión crítica de la realidad y una participación real de los educandos en el proceso pedagógico, con el fin de transformar la realidad social local (Ocampo, 2008).

Según Ocampo (2008), este paradigma parte de la premisa según la cual existe una relación de opresión entre clases sociales, e invita a los oprimidos a transformar la realidad social, a través de la práctica, por medio de procesos educativos que permitan tener conciencia de la realidad social, así como adquirir recursos sociales y políticos para transformar la relación de opresión. Desde esta perspectiva es pertinente orientar la construcción de las metodologías desde y con las comunidades, con el fin de generar procesos dialógicos de concientización crítica sobre las afectaciones del conflicto armado. Con esto, se podrán fortalecer escenarios de transformación, desde la potencialización de los recursos propios jurídicos y psicosociales de las víctimas que participan en la JEP.

Al respecto, para el desarrollo de las estrategias es necesario incorporar elementos metodológicos que les permitan a las víctimas interiorizar los saberes que se pretenden construir. Para esto, es conveniente "traducir" el lenguaje jurídico de los contenidos tratados a un lenguaje que pueda ser significativo para la comunidad que participa. Así, con orientación en la didáctica, Posada (2014) propone el juego como herramienta metodológica que permite una relación dinámica en pro de la transmisión de saberes y aporta a la reflexión de lo enseñado y aprendido. Además, la herramienta del juego permite evocar las experiencias pasadas de los participantes para, así, lograr un aprendizaje significativo de los contenidos que se pretende enseñar. 
Finalmente, de acuerdo con lo anterior, es conveniente diseñar metodologías creativas que posibiliten un desarrollo cómodo y empático de las actividades. Para la didáctica, el objetivo es transmitir saberes desde una forma descriptiva y creativa - tanto el enseñar como el aprender-. En ella cobra importancia la acción pedagógica que busca la promoción de relaciones dinámicas entre quienes conforman el escenario de enseñanza-aprendizaje, con base en los significados que convergen en las nuevas formas de conocimiento. En dicha convergencia, desde la didáctica, el disfrute y el desafío son elementos fundamentales.

\section{Método}

\section{Diseño metodológico}

La construcción de esta caja de herramientas se llevó a cabo en el marco de las metodologías participativas (Francés, Alaminos, Penalva y Santacreu, 2015), las cuales se pueden entender como procesos, de investigación e intervención transversalizados por la idea de que todos los actores involucrados en el proceso deben participar decididamente en este.

Las metodologías participativas están enmarcadas en el paradigma de los métodos cualitativos e involucran componentes de distintos tipos que se requieren para el planteamiento metodológico de cuatro componentes. Los politico-institucionales facilitan la gestión de interacciones entre representantes de las instituciones y de la sociedad civil, así como la difusión de información. Los componentes contextuales tienen en cuenta el tiempo, las experiencias previas y los límites legales que tienen los procesos. Los componentes operacionales definen el grado de participación y deliberación, la construcción de consensos, la reflexión-acción y evaluación de los procesos. Finalmente, los componentes actitudinales implican la necesidad de ciertos comportamientos en quienes llevan a cabo la intervención o la investigación (Francés et al., 2015). Estos componentes son aspectos que fueron tenidos en cuenta a la hora de diseñar las metodologías. 


\section{Técnicas para la recolección de información}

A continuación, se describen las técnicas propuestas para cada uno de los elementos que se desarrollaron. La interrelación entre ellas se expondrá en el apartado "Fases".

\section{Sistematización de experiencias}

La sistematización de experiencias es una herramienta que permitió analizar varias experiencias en las metodologías que se aplicaron, con el fin de descubrir o hacer explícitas las lógicas y dinámicas que intervinieron en el proceso, así como los factores internos o externos que han influido en su desarrollo (Expósito y González, 2017). Según Expósito y González (2017),

[...] se concibe la sistematización como la reconstrucción y reflexión analítica de una experiencia mediante la cual se interpreta lo sucedido para comprenderlo; por lo tanto, esta permite obtener conocimientos consistentes y sustentados, comunicarlos, confrontar la experiencia con otras y con el conocimiento teórico existente, y así contribuir a una acumulación de conocimientos generados desde y para la práctica.

\section{Observación participante}

Esta técnica permitió adquirir información sobre el mundo social de la población con la participación de los facilitadores. A través de esta observación, los datos se pudieron sistematizar desde un proceso reflexivo, analítico e interpretativo, tanto de la corporeidad como del lenguaje relacional de cada participante, en circunstancias determinadas. Aquí, los facilitadores mantienen un compromiso concreto para realizar acciones participativas dentro de las actividades con las comunidades (Scribano, 2008).

Para llevar a cabo la observación participante, se utilizaron diarios de campo, instrumentos que permitieron registrar aquellos hechos de relevancia que son interpretados según lo observado. Los diarios de campo dieron cuenta de la conexión 
sobre la percepción y subjetividad de cada facilitador, lo que determinó los elementos predominantes y significativos de la dinámica interaccional, como de los hechos relevantes que se manifestaron durante la aplicación de la técnica.

\section{Revisión documental}

Esta técnica permitió identificar investigaciones, textos y discusiones, elaboradas con anterioridad, sobre experiencias metodológicas en procesos de construcción de paz y de derechos humanos. Con ella es posible construir premisas de partida y establecer semejanzas, diferencias y conexiones entre textos, además de precisar ámbitos no explorados (Valencia, 2015, p. 3).

\section{Análisis de información}

Para la construcción de la caja de herramientas, se analizó la información recopilada a partir de los criterios del análisis categorial, una técnica que organizó la generación de información proveniente de múltiples fuentes. Dicho orden se establece mediante la agrupación y clasificación de datos para los respectivos análisis y es flexible en la medida en que permite el análisis de material no estructurado (Galeano y Aristizábal, 2008). gracias a esta técnica fue posible comprender distintos componentes que deben ser incluidos a la hora de construir herramientas que integren la caja. El análisis categorial se elaboró a través de una matriz que tuvo como fin sistematizar la información recolectada en cada fase metodológica, y permitió identificar, por medio de un proceso analítico, los resultados que emergen de la interpretación general de las categorías correspondientes.

\section{FASES}

La construcción de la caja de herramientas se dividió en varias fases. Vale la pena aclarar que la ejecución de las fases no debe entenderse en un sentido lineal, debido a que cada una de ellas puede alimentar la construcción de la siguiente $y$, al mismo 
tiempo, realimentar la fase anterior, en un proceso cíclico. Las fases se representan en la figura 3.1:

Figura 3.1. Fases metodológicas.

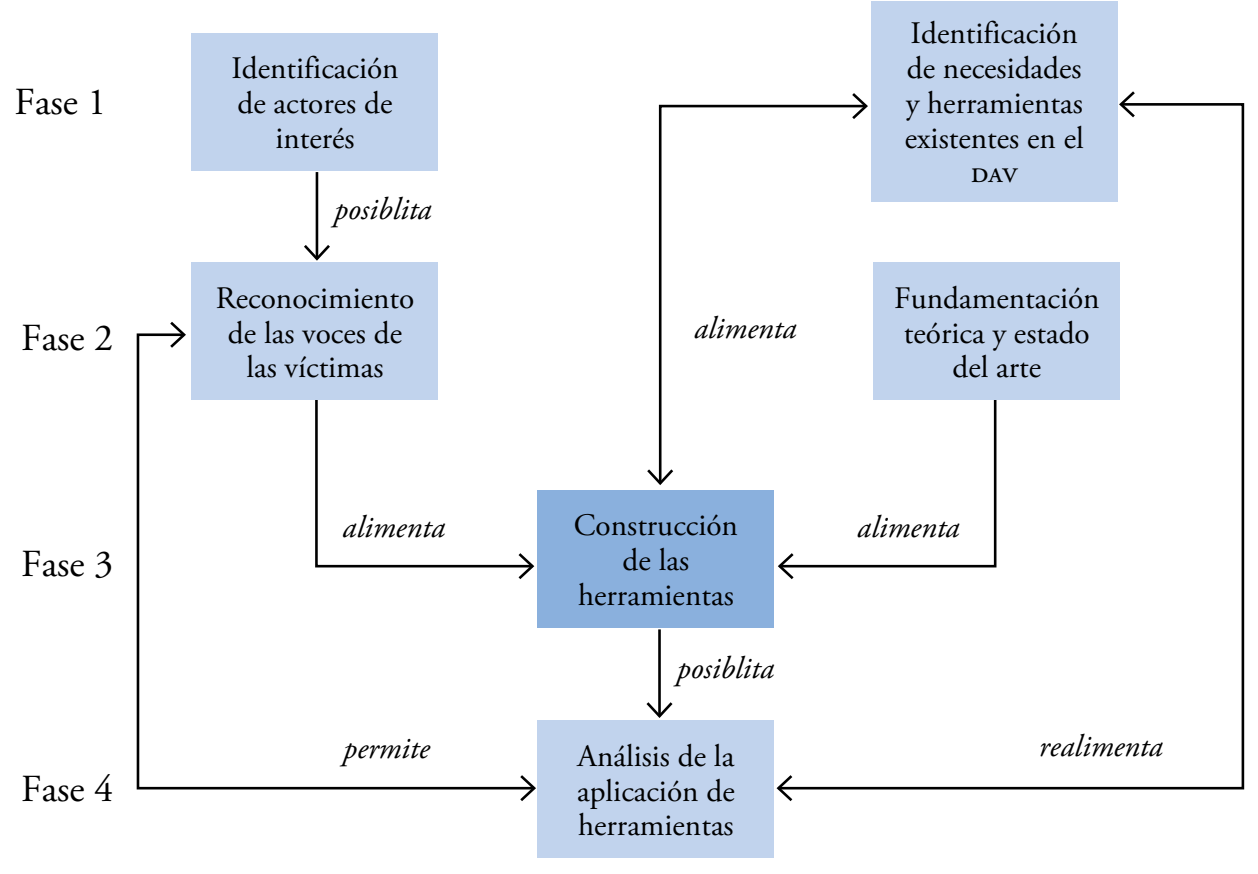

Fuente: elaboración propia. 
Para desarrollar estas fases con sus respectivos elementos, se implementaron las técnicas que se muestran en la tabla 3.1.

Tabla 3.1. Técnicas propuestas para los elementos y las fases de la caja de herramientas

\begin{tabular}{lll}
\multicolumn{1}{c}{ Fases } & \multicolumn{1}{c}{ Elementos } & \multicolumn{1}{c}{ Técnicas } \\
\hline Fase 1 & Identificación de actores & Revisión documental \\
\hline Fase 2 & $\begin{array}{l}\text { Reconocimiento de las voces } \\
\text { de las víctimas }\end{array}$ & $\begin{array}{l}\text { Observación participante y sistematización } \\
\text { de experiencias }\end{array}$ \\
\hline Fase 3 & Revisión teórica & Revisión documexntal \\
\hline Fase 4 & $\begin{array}{l}\text { Aplicáno de las herrán y análisis de las } \\
\text { herramientas aplicadas }\end{array}$ & $\begin{array}{l}\text { Revisión documental, observación participante } \\
\text { y sistematización de experiencias }\end{array}$ \\
\hline & $\begin{array}{l}\text { Observación participante y sistematización } \\
\text { de experiencias }\end{array}$
\end{tabular}

Fuente: elaboración propia.

En relación con la figura 3.1 y la tabla 3.1, en la Fase 1 se revisó el documento Borrador de la estrategia de acompañamiento y orientación psicojurídica del DAV (Departamento de Atención a Víctimas, 2019a) y el Plan Operativo Anual del DAv (Departamento de Atención a Víctimas, 2019b). Con esta revisión se identificaron cuáles son los actores a los que se dirigen las acciones del Departamento y cuáles son las necesidades que se planean satisfacer a partir de la construcción de esta caja de herramientas.

En la Fase 2, se realizó una revisión documental de los autores y fuentes consultadas en la fundamentación teórica presentada anteriormente, lo cual permitió identificar algunos aspectos teóricos que fueron tenidos en cuenta en la construcción de las estrategias metodológicas. Además, con el fin de encontrar orientaciones metodológicas para la construcción de las estrategias, se revisó el libro Recordar y narrar el conflicto (Centro Nacional de Memoria Histórica y University of British Columbia, 2013); La investigación participativa (Francés et al., 2015); el documento 
Modelo pedagógico para la educación en derechos humanos (Robayo, 2014); el libro Juegos para actores y no actores (Boal, 2001), que sirvió como insumo metodológico para utilizar técnicas del Teatro del Oprimido, y el Guion metodológico para entrevista semiestructurada Villapaz y el virus del olvido (Amorocho, Báez, Botero, Tovar y Ricardi, 2018), que fue utilizado como insumo para la construcción de metodologías para el trabajo con niños, niñas y adolescentes.

También para esta fase se sistematizaron las experiencias del taller reflexivo La Ciudad de la JEP — en el que participaron 33 personas del Movimiento de Víctimas de Crímenes de Estado (Movice) — y del taller reflexivo El Pueblo de la JeP —en el que participaron 7 personas de la Mesa de Participación de Víctimas de Mosquera-, con el fin de recoger las opiniones que habían tenido los participantes en relación con la construcción y la implementación de las metodologías. La experiencia de haber podido covalidar y coconstruir las metodologías con las víctimas permitió identificar elementos diferenciales de mucha importancia. Estos incluyen que las metodologías deben ser flexibles al hecho victimizante, a los enfoques diferenciales (genero, discapacidad, étnico, racial, curso de vida) y a un enfoque territorial, pues se reconoce que no es lo mismo desarrollar metodologías con colectivos u organizaciones de víctimas ubicadas en zonas urbanas que con víctimas ubicadas en las zonas rurales del país. Por tanto, en la Fase 3 se construyeron las herramientas a partir de la información obtenida en las fases anteriores. Por último, en la Fase 4 se sistematizó la información y se obtuvieron conclusiones a partir de la implementación de los guiones metodológicos que se construyeron. Esta información sirvió para retroalimentar la formulación de las estrategias metodológicas propuestas.

\section{RESULTADOS Y DISCUSIÓN}

Tras analizar la información seleccionada bajo los criterios del análisis categorial, para cada una de las fases y sus respectivos elementos, se reconocieron algunos aspectos para tener en cuenta en el diseño, que dieron como resultado los siguientes guiones metodológicos: 


\section{De alistamiento: presentación del Sistema y la JEP, y presentación de la estrategia psicojurídica}

\section{La Ciudad de la JEP}

Promueve la comprensión acerca de la Jurisdicción Especial para la Paz y posibilita un conocimiento interinstitucional, pues plantea el desarrollo de un taller de orden lúdico-pedagógico en el que se explican los diferentes procesos y dinámicas que ocurren dentro de la JEP, por medio de representaciones visuales alusivas a una ciudad (peajes, caminos, edificios, parques, etc.). El taller está dividido en estaciones, en las cuales los participantes conocen la JEP por medio de actividades lúdicas, guiadas por los facilitadores. En cada una de las estaciones, los participantes llevan a cabo una actividad particular.

\section{Coconstrucción de la "Estrategia de acompañamiento y orientación psicojurídica"}

Genera un escenario de socialización, que permita llegar a reflexiones y discusiones alrededor de la "Estrategia de acompañamiento y orientación psicojurídica", si esta es adecuada para implementarse en los territorios, si el lenguaje utilizado es el apropiado y si logra cumplir con las expectativas de las víctimas. En esta actividad se incorporan elementos y técnicas del Teatro del Oprimido (Boal, 2001) y de asociación de palabras, que permiten a la comunidad ser reconocida como protagonista y actuante en los procesos que se llevan a cabo en esta estrategia.

\section{De fortalecimiento: justicia transicional y justicia restaurativa}

\section{Parqués Especial para la Paz}

Posibilita la construcción de espacios de difusión e información, en este caso enmarcados en las comprensiones de la justicia transicional y la justicia restaurativa a través de la analogía del juego de mesa Parqués, mediante la cual se representan medidas alternativas de justicia que posibilitan profundizar en la deconstrucción de la imagen del "enemigo". 


\section{De evaluación: encuesta participativa}

\section{Cuestionario Participativo de Satisfacción respecto al Acompañamiento Psicojurídico}

Permite alcanzar dos objetivos. Por un lado, — como cualquier encuesta - obtiene información cuantitativa significativa sobre las impresiones de las víctimas de los procedimientos judiciales en los que participan. Por otro lado, permite un grado de reflexión colectiva y de autoconocimiento, en tanto que es la población quien da cuenta de los contenidos que deben tratarse en el formulario y cuál debe ser la interpretación de los resultados, lo que permite un análisis y priorización de los temas que les afectan (Francés et al., 2015).

\section{Diferencial: enfoque de niñas y niños, y territorial}

\section{Villa Paz y el Virus del Olvido (adaptación)}

Es una adaptación de la metodología titulada "VillaPaz y el Virus del Olvido" (Amorocho, Báez, Botero, Tovar y Riccardi, 2018), en la cual se representa un cuento, mientras se ahonda en cómo se vive la paz en tres ambientes diferentes: familia, colegio y comunidad; luego, un último ambiente consiste en reflexionar sobre cómo cada uno puede ser agente de paz. La metodología se ha orientado por unas estaciones en las que, mediante herramientas artísticas, se tiene la intención de visibilizar la integración de significados de justicia y verdad en torno a la construcción de paz. El resultado final es un librillo para las niñas y los niños que consigna las diferentes apuestas artísticas desarrolladas por sí mismos durante el desarrollo de la jornada.

\section{El Pueblo de la JEP}

Al igual que el guion de La Ciudad de la JEP, este promueve la comprensión acerca de la Jurisdicción Especial para la Paz y posibilita un conocimiento interinstitucional, pues plantea la realización de un taller de orden lúdico-pedagógico en el que se explican los diferentes procesos y dinámicas que ocurren dentro de la JEP, por medio 
de representaciones visuales alusivas a un pueblo (montañas, caminos, plazas, trochas, etc.). También se encuentra dividido en estaciones, en las cuales los participantes conocen la JEP por medio de actividades lúdicas, guiadas por facilitadores. En cada una de las estaciones, los participantes llevan a cabo una actividad particular.

Como resultado de la experiencia de prácticas, se destaca que las metodologías propuestas son coherentes con los marcos teóricos que cobijaron su construcción por tres razones. En primer lugar, tienen en cuenta las dimensiones relacionales y contextuales de las comunidades, lo cual es coherente con el enfoque de la psicología sistémica. En segundo lugar, fue posible que se reconocieran los saberes de estas comunidades, y los relacionan con los contenidos que se quieren tratar en los talleres propuestos, lo que responde a los preceptos de la psicología social comunitaria y de la educación popular. En tercer lugar, estas metodologías contribuyen a la transformación de la realidad social, a partir del fortalecimiento de las capacidades de las víctimas para el acceso a derechos, lo cual coincide con elementos de la psicología social comunitaria y de la psicología de la liberación.

Estas perspectivas resultaron pertinentes parala construcción de una caja de herramientas puesto que, desde el punto de vista metodológico, invitaron al manejo del lenguaje en la construcción y transformación de realidades, a partir de una postura dialógica con los participantes, tras un reconocimiento contextual e histórico. Lo anterior llevó a orientar la relación de los profesionales que van a llevar a cabo metodologías, a través de premisas que se deben tener en cuenta en las acciones psicosociales, que implican la comprensión del otro a través del entendimiento de sus relaciones.

Estas corrientes teóricas posibilitaron la comprensión local de las problemáticas de las comunidades a las cuales están dirigidas las metodologías; por tanto, es conveniente diseñar metodologías creativas que posibiliten un desarrollo cómodo y empático de las actividades. A su vez, desde una mirada interrelacional, promovieron la incorporación de saberes populares durante la implementación de las herramientas, lo que estimuló el uso de métodos verdaderamente participativos. También se reconoció la necesidad de que, en el diseño, la ejecución y la evaluación de estas estrategias se tenga como fin contribuir al fortalecimiento de capacidades jurídicas y psicosociales, de manera que 
se aporte en la concientización de las afectaciones del conflicto armado, para que las comunidades logren transformar en alguna medida su realidad social, a través de su participación en la Jurisdicción.

En cuanto a los logros, en la implementación de los talleres se identificó que las propuestas metodológicas lograron establecer relaciones de confianza entre la entidad y las comunidades, lo que posibilitó nuevos escenarios de contacto. Además, se lograron identificar las opiniones que tenían las víctimas respecto a la jurisdicción, y se explicaron de manera clara conceptos jurídicos y psicosociales, de tal manera que fuera interiorizada por los participantes. Asimismo, algunas de las metodologías lograron que el uso de metáforas contribuyera para que emergieran nuevas narrativas relacionadas con la justicia y la reparación.

En relación con las recomendaciones, la principal de ellas tiene que ver con el manejo del tiempo a la hora de llevar a cabo los guiones metodológicos. Por otro lado, se recomienda tener en cuenta los enfoques diferenciales en el desarrollo de las actividades, especialmente para aquellas personas que están en condición de alguna discapacidad física o motriz.

En cuanto a las limitaciones de los guiones metodológicos propuestos, es relevante mencionar, primero, que el enfoque diferencial étnico no se pudo implementar en plenitud, puesto que este requiere de conocimientos y experiencia relacionada con la cosmovisión de las comunidades étnicas que se acompañen; por esto, necesitan de metodologías que respondan específicamente a su contexto espiritual y cultural. A su vez, se reconoce también la posibilidad de que, en algunos contextos, las metodologías no puedan implementarse en plenitud - debido a cuestiones de tiempo o de lugar-. Sin embargo, los contenidos, las preguntas orientadoras y las técnicas que se utilizan pueden ser útiles para la construcción y adaptación de nuevas metodologías, con el fin de fortalecer las capacidades de las víctimas en su proceso de participación en distintos escenarios, para que así se contribuya con la satisfacción de sus derechos.

En efecto, la experiencia de prácticas que se expone aquí contribuye a satisfacer una necesidad de la JEP de promover espacios para la participación de las víctimas (art. 
14 de la Ley Estatutaria). Además, aporta en el cumplimiento de las medidas para la participación de las víctimas: acceso a información, asistencia jurídica y psicosocial (punto 5 del Acuerdo Final), y responde a la necesidad coyuntural e histórica de que la psicología participe y contribuya en la reconstrucción del tejido social, en la superación de la violencia, en el restablecimiento de los derechos de las víctimas y en la construcción de paz.

\section{Conclusiones}

Para terminar, se insiste en que los guiones metodológicos expuestos responden a las necesidades de acompañamiento y pedagogía del Departamento de Atención a Víctimas (DAv). Producto de la práctica profesional, surgieron las siguientes recomendaciones metodológicas y psicosociales para la construcción de metodologías en el marco de la JEP:

- Las metodologías deben ser flexibles ante el hecho victimizante, los actores, los enfoques diferenciales y territoriales.

- Es necesario que las metodologías incorporen aspectos simbólicos y espirituales de las comunidades.

- Es necesario que las metodologías permitan informar acerca de los avances que se han dado en la jurisdicción.

- El desarrollo de las metodologías debe invitar al manejo atento del lenguaje, pues la realidad es una construcción social mediada por este, lo que permite la creación y transformación de realidades.

- Las metodologías deben orientar el accionar de los equipos psicojurídicos a través de premisas que implican la comprensión del otro, mediante el entendimiento de su contexto y de sus relaciones.

- Las metodologías deben apostarle a promover el uso de métodos verdaderamente participativos. 


\section{REFERENCIAS}

Agudelo, M. y Estrada, P. (2012). Constructivismo y construccionismo social: algunos puntos comunes y algunas divergencias de estas corrientes teóricas. Prospectiva, 17, 353-378.

Amorocho, Báez, Botero, Tovar y Ricardi. (2018). Guion metodológico Villa Paz y el Virus del Olvido [documento inédito]. Universidad de la Sabana, Chía, Colombia.

Anderson, H. (1997). Conversación, lenguaje y posibilidades. Buenos Aires: Amorrortu.

Boal, A. (2001). Juego para actores y no actores. Barcelona: Alba Editorial; sıu.

Centro Nacional de Memoria Histórica y University of British Columbia. (2013). Recordar y narrar el conflicto: herramientas para reconstruir memoria histórica. Bogotá: Imprenta Nacional. Consultado en http://www.centrodememoriahistorica.gov.co/descargas/informes2009/recordar-narrar-el-conflicto.pdf.

Congreso de Colombia. (2019, 6 de junio). Ley Estatutaria para la Administración de Justicia en la Jurisdicción Especial para la Paz [Ley 1957]. Diario Oficial n. ${ }^{\circ}$ 50.976. Consultado en http://www.suin-juriscol.gov.co/viewDocument.asp?id=30036543.

Departamento de Atención a Víctimas. (2019a). Documento borrador de la Estrategia de Acompañamiento y Orientación Psicojurídica [documento inédito]. Jurisdicción Especial para la Paz. Bogotá, Colombia.

Departamento de Atención a Víctimas. (2019b). Plan operativo anual del Departamento de Atención a Víctimas [documento inédito]. Jurisdicción Especial para la Paz. Bogotá, Colombia.

Expósito, D. y González, J. (2017). Sistematización de experiencias como método de investigación. Gaceta Médica Espirituana, 19(2). Consultado en http://revgmespirituana.sld. cu/index.php/gme/article/view/1497/html. 
Francés, F. J., Alaminos, A., Penalva, C. y Santacreu, O. A. (2015). La investigación participativa: métodos y técnicas. Cuenca: Pydlos Ediciones. Consultado en http://dspace. ucuenca.edu.ec/bitstream/123456789/23455/1/Libro\%20LA\%20INVESTIGACI\%20 PARTICIPATIVA\%20repositorio.pdf.

Freire, P. (1968). Pedagogía del oprimido. Madrid: Siglo XXI.

Galeano, M. N. y Aristizábal, M. E. (2008). Cómo se construye un sistema categorial. Estudios de Derecho, 65(145). Consultado en https://revistas.udea.edu.co/index.php/red/ article/view/848.

Gobierno de Colombia y Farc-ep. (2016, 24 de noviembre). Acuerdo Final para la terminación del conflicto y la construcción de una paz estable y duradera. Consultado en https:// www.unidadvictimas.gov.co/sites/default/files/documentosbiblioteca/nuevoacuerdofinal.pdf.

Martín-Baró, I. (1998). Hacia una psicología de la liberación. En Psicología de la liberación. Madrid: Editorial Trotta.

Medina, M. V., Layne, B., Galeano, M. P. y Lozada, C. (2007). Lo psicosocial desde una perspectiva holística. Tendencias \& Retos, 12, 177-189.

Montero, M. (2003). Psicología social crítica (Editorial). Revista Interamericana de Psicología, $37(2), 1-3$.

Montero, M. (2004). Relaciones entre psicología social comunitaria, psicología crítica y psicología de la liberación: una respuesta latinoamericana. Psykhe, 13(2), 17-28. Consultado en http://www.catedralibremartinbaro.org/pdfs/Relaciones-Entre-Psicologia-SocialComunitaria-Psicologia-Critica-y-Psicologia-de-la-Liberacion-U.pdf.

Ocampo, J. (2008). Paulo Freire y la pedagogía del oprimido. Revista Historia de la Educación Latinoamericana, 10, 57-72. 
Oficina del Alto Comisionado para la Paz. (s. f.). Información relacionada con el Acuerdo Victimas. Consultado el 10 de marzo de 2019, en http://www.altocomisionadoparalapaz.gov. co/Documents/informes-especiales/abc-del-proceso-de-paz/abc-jurisdiccion-especialpaz.html.

Posada, R. (2014). La lúdica como estrategia didáctica (tesis doctoral). Universidad Nacional de Colombia, Bogotá.

Robayo, G. A. (2014). Modelo pedagógico para la educación en derechos humanos. Bogotá: Defensoría del Pueblo.

Scribano, A (2008). La observación. En El proceso de investigación social cualitativo. Buenos Aires: Prometeo.

Stanton, M. y Welsh, R. (2012). Systemic thinking in couple and family psychology research and practice. Couple and Family Psychology: Research and Practice, 1(1), 14-30.

Valencia, V. (2015). Revisión documental en el proceso de investigación. Unvirtual. Universidad Tecnológica de Pereira, Colombia. Consultado en https://bit.ly/2QSzPx4. 